\title{
THE APPLICATION OF MICRONIZATION TECHNOLOGY ON A FRUITING BODY EXTRACT OF ANTRODIA CINNAMOMEA
}

\author{
Chih-Cheng Lin ${ }^{1}$, Hung-Kai Yen ${ }^{1}$, Shun-Hsiang Weng ${ }^{2}$ \\ ${ }^{1}$ Department of Biological and Technology, Meiho University, 23, Pingguang Rd., Neipu, Pingtung, \\ Taiwan, R.O.C. \\ ${ }^{2}$ Department of Food Science and Nutrition, Meiho University, 23, Pingguang Rd., Neipu, Pingtung, \\ Taiwan, R.O.C. \\ هkyen@meiho.edu.tw \\ https://doi.org/10.34302/crpjfst/2020.12.5.7 \\ Article history: \\ Received: \\ 29 August 2020 \\ Accepted: \\ 25 December 2020 \\ Keywords: \\ Food dispersant; \\ Antrodia cinnamomea; \\ Micronizer.

\begin{abstract}
Micronization has been applied in many drug preparations-including traditional Chinese medicine-to optimize oral bioavailability. The commonest technique used is mechanical commination (e.g., by crushing, grinding, and milling). Particles produced through the micronization process are quite easy to re-aggregate due to the Van der Waals effect, and therefore dispersants are added to maintain the stability of the micronized mixture and prevent them from aggregating into larger particles. In this study, the particles of Antrodia cinnamomea suspended in water extract are micronized by the impact between two currents of liquid generated by a liquid micronizer made specifically for the task. Antrodia cinnamomea fruiting bodies were extracted using $95 \%$ alcohol. The extraction was then diluted 20 times with water and underwent a micronization process for 240 minutes. Appropriate amounts of dispersant were then added to the micronized Antrodia cinnamomea extract, with each dispersant divided into three concentrations $-0.5 \%, 0.75 \%$, and $1.0 \%$. Three different dispersants were tested in this study, namely microcrystalline cellulose, silicon dioxide, and polyethylene glycol. Monitoring of the degree of aggregation over 56 days showed that a silicon dioxide concentration of $0.75 \%$ gives the best
\end{abstract} \\ dispersion effect.
}

\section{Introduction}

Antrodia cinnamomea (Syn. Antrodia camphorata) is a fungal parasite found only on the inner cavity of the endemic species Cinnamomum kanehirae (Bull camphor tree) Hayata (Lauraceae) (Tzeng et al., 2011). Due to its rareness, the price of Antrodia cinnamomea varies from tens of thousands to hundreds of thousands of US dollars per kilogram in the market, which is why it is regarded as the most expensive wild fungus in the world. Taiwanese indigenous people first found the fruiting body of this species in their search for wood, and after trying it and drink its boiled juice, they noticed that the fungus could relieve hangovers, eliminate fatigue, and improve liver disease. As a result, Antrodia cinnamomea has long been used as a medical treatment for food poisoning, diarrhea, hepatitis, high blood pressure, etc., by indigenous people in Taiwan. Antrodia cinnamomea is also a potential ingredient in modern health supplements and pharmaceuticals thanks to its antioxidant, nitrite scavenging, and antitumor properties (Cha et al., 2009).

Antrodia cinnamomea contains various bioactive compounds, such as terpenoids, benzenoids, lignans, benzoquinone derivatives, and succinic and maleic derivatives, in addition to polysaccharides. Polysaccharides can 
improve the human immune response and inhibit the hepatitis B virus (Lee et al., 2002), while triterpenoids provide mainly anti-cancer and liver protection (Laszczyk et al., 2009), and superoxide dismutase (SOD) eliminates free radicals. However, extracting bioactive compounds is not easy due to their low solubility, and the adsorption rate in the human body is not ideal. Therefore, to optimize the adsorption, many methods have been applied, such as through Self-Emulsifying Drug Delivery Systems (SEDDS) (Tang et al., 2007), micronization (Xu et al., 2004), etc. In this experiment, the micronization method was applied. In recent years, micronization has been applied in numerous fields, from foodstuffs to pharmaceuticals, and especially in traditional Chinese medicine. In addition to being effective in terms of improving its bioavailability, the micronization process can also reduce the side effects of traditional Chinese medicine (Fan et al., 2019). Moreover, the amount of raw materials used can be reduced, thus achieving higher economic value. For example, the physiological effects of dietary fibers are related to their absorption, so increasing the specific surface area can improve the physiological activity of dietary fibers. By reducing the particle size of dietary fibers from $0.1 \mathrm{~mm}$ to 1 $\mu \mathrm{m}$, the specific surface area can be increased by a factor of 100, and the recommended intake can be reduced to $1 \%$ of the original one. If the particle size is further reduced to 100 nanometers, the recommended intake can be reduced to one thousandth compared to the original (Lin et al., 2012). However, the smaller the particles, the easier the re-aggregation. According to DLVO theory, there is a mutual attraction between colloidal particles (the Van der Waals force), and there is also a mutual repulsive force, which is the electrostatic repulsive force when the electric double layers overlap. These two opposing forces determine the stability of the colloid. When the attractive force between the particles is dominant, small particles are easy to aggregate and precipitate, because the attraction force causes the continuous aggregation phenomenon. When the electrostatic repulsion force is dominant, the particles can be prevented from aggregation and precipitation, so the colloids will be in a stable state. In many cases, in order to obtain substances with different stabilities, researchers will increase or decrease the attraction or repulsion via environmental changes. Common methods include changing the ionic environment, adjusting the $\mathrm{pH}$ value, or adding surface-active substances (polymeric dispersants), and so on (Alwadani et al., 2018). The factors and parameters that affect aggregation need to be set according to different substances, such as temperature, humidity, concentration, $\mathrm{pH}$ value, pressure, electrical potential, etc. (Zapadka et al., 2017). The empirical results of this study show that the original high-particle concentration with a particle size of less than $1 \mu \mathrm{m}$ accounted for $69 \%$ of the suspension. After storage at $4{ }^{\circ} \mathrm{C}$ for 24 hours, the average particle size increased from $0.79 \mu \mathrm{m}$ to $9.35 \mu \mathrm{m}$. After thawing, the average particle size was $109 \mu \mathrm{m}$, reaching $132 \mu \mathrm{m}$ after high-temperature sterilization. This means that there is an absolute correlation between temperature and the degree of reaggregation. The lower the temperature, the more severe the reaggregation. The concentration of particles is an important factor that affects the degree of reaggregation and thus the stability of the liquid. The higher the concentration, the higher the probability of collision between the particles, meaning that the aggregation phenomenon is relatively proportional. To modify the suitable interface of the materials before applying it to the final product, dispersants can be added in order to make the scattered targets become well distributed, thus stabilizing them in the medium. Depending on the actual requirements and applications, each type of product has a different definition of stability. For example, in some fields, if a colloidal mixture is stable for two days, it is considered stable, but in others, it may need to be stable for more than two years without obvious changes in order to be accepted as such. In this study, three dispersants were used: microcrystalline cellulose (MCC), silicon dioxide $\left(\mathrm{SiO}_{2}\right)$, and polyethylene glycol. MCC 
is a pure natural cellulose, a product made by converting fibrous cellulose into a re-dispersible gel or aggregate of crystalline cellulose. This material can be dried into pure fine particles and made into powder (Mikaela Börjesson, 2015). The color of MCC powder is white, and the powder is odorless and tasteless, which is why it is commonly used in pharmaceuticals, health foods, and cosmetics. Silicon dioxide $\left(\mathrm{SiO}_{2}\right)$ - an acidic oxide-exists in two main types as crystalline and amorphous. Amorphous silicon dioxide has been used in food applications for many years, such as in beer and wine, and as an anti-caking agent in powder (Guo et al., 2018) and thick paste products. Polyethylene glycol is an oligomer or polymer of ethylene oxide, which vary in molecular weight. Different molecular weights in different applications often have different physical properties (such as viscosity, etc.) (Vidyasagar et al., 2016), but most polyethylene glycols are similar in terms of their chemical properties. Polyethylene glycol 400 (PEG400) was chosen for this experiment due to its stability and due to the fact that it does not deteriorate easily (it has also been used widely in various industries).

\section{Materials and methods}

\subsection{Antrodia cinnamomea extraction}

Since the growth speed of Antrodia cinnamomea is slow, the most suitable cultivation conditions for it are artificial cultivation. Besides chemical factors (such as carbon and nitrogen sources in the medium), physical factors also play a crucial role in the cultivation of the fungus, especially temperature. Different strains will have different discrepancies, and the most suitable temperature for the mycelium in submerged culture is between 22 to $28^{\circ} \mathrm{C}$. Exceeding this temperature can cause the mycelium to age more easily (Hadar and Dosoretz, 1991). Humidity is also a key factor affecting the cultivation of Antrodia cinnamomea, with a range between $40 \%$ and $80 \%$ being the most suitable. Physical wounds and the concentration of gas can also affect the growth rate of fruiting bodies. Furthermore, since a source of carbon and nitrogen is a very important factor in cultivating mushrooms, these elements are indispensable in the Antrodia cinnamomea culture medium. The carbon to nitrogen ratio $(\mathrm{C} / \mathrm{N})$ also affects the content of the components of the fungus. Commonly used sources of carbon are glucose, fructose, sucrose, maltose, etc. The concentration of glucose in the medium was $3 \%$, which is the most suitable carbon source for mycelium. When the concentration of yeast extract reaches $3 \%$, the biomass and the yield of triterpenes increase. The optimal conditions are 3\% of yeast extract, $1 \%$ of peptone, and $2.5 \%$ of malt extract (Chang et al., 2006). The source of nitrogen is the main source of mushroom protein and nucleic acid. Different carbon sources have different effects on mycelial growth (Sone et al., 2014). Glucose (Wanglaichang Co., Ltd), malt extract (Sisco Research Laboratory Pvt), agar and peptone (Yihe Co., Ltd) were used to prepare the medium. Antrodia cinnamomea mycelium was obtained by isolating the mycelium in a solid medium (3\% glucose, $2 \%$ malt extract, $2.5 \%$ agar). To prepare the submerged culture, 3\% glucose, $2 \%$ malt extract, and $1 \%$ peptone were combined in an Erlenmeyer flask. After transferring the mycelium into the new medium, the flask mouth was wrapped with parafilm and the flask was placed on a shaker (TS520D model, Yihder Technology) for 14 days at 130 rpm. Cinnamomum kanehirae can be found on the Jiaoban Mountain in the Taoyuan region, in Nanzhuang of the Miaoli region, in Zhushan of the Nantou region, and in Liugui of the Kaohsiung region of Taiwan (Lin et al., 2006). The wood of the Cinnamomum kanehirae is the sole host, mainly because its tolerance to the essential oil of Antrodia cinnamomea is relatively high, so other fungi cannot grow on it (Geethangili and Tzeng, 2011). A stout camphor $\log$, used to culture the Antrodia cinnamomea fruiting body, was sterilized for two hours in an autoclave (HY-3005V model and HY230 model, Hungyi Instrument Co., Ltd) and then exposed to UV light for the next 24 hours. The culturing steps were performing in the laminar airflow bench (Hae Tian Science Co., Ltd): the mycelium-contained in the liquid medium-was spread throughout the chilled log and incubated inside a thermostatic chamber at $26^{\circ} \mathrm{C}$ to $28^{\circ} \mathrm{C}$. The Antrodia cinnamomea 
fruiting body formed after eight to ten months. To make the Antrodia cinnamomea aqueous extract, one kilogram of Antrodia cinnamomea fruiting body was cleaned thoroughly with sterile water and dried in the shade before being combined with 20 liters of $95 \%$ ethanol (Pingtung Brewery Co., Ltd) and settled in a glass bottle for one month at room temperature. Ethanol in the mixture was then evaporated completely. The Antrodia cinnamomea extract was diluted twenty times with distilled water to obtain a mixture, here called Antrodia cinnamomea liquid (ACL).

\subsection{Micronizing the Antrodia cinnamomea liquid (ACL)}

The micronizer used in this study was designed according to the impact principle, which states that particles suspended in liquid can be micronized by creating two contrary liquid flows. When the machine is operated at high speed, the interior of the machine will activate a gas circulation function. The machine consists of a round mixing tank with two stirring assemblies inside, a thermometer, a liquid inlet and outlet, and four stands [Fig. 1. (a)]. On the tank lid, there are three different observation holes as well as a pressure valve, an exhaust vent, and a liquid inlet [Fig. 1. (b)]. Each stirring assembly comprises a high-speed motor (which controls the flow direction), a shaft, and a crossshaped stirring blade. The first motor operates clockwise while the other one does so counterclockwise, which causes the impact between two flows of liquid. Two stirring assemblies are arranged in a parallel and equidistant position inside of the mixing tank. To micronize the ACL (ACL240), the liquid was added through the inlet and beaten continuously for 240 minutes.
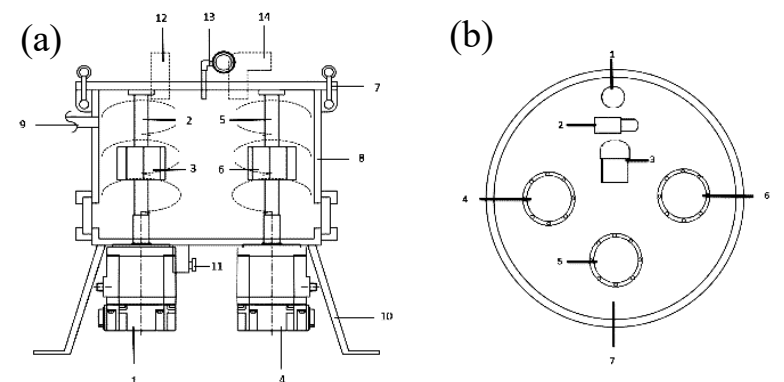

Figure 1. (a) schematic of the nanometerization machine (1, 4: high-speed motors; 2,5 : shafts; 3 ,
6: stirring blades; 7: lid; 8: tank body; 9: thermometer; 10: stands; 11: outlet; 12: inlet; 13: pressure valve; 14: exhaust vent. (b) plan view of the nanometerization machine (1: inlet; 2: pressure valve; 3: exhaust vent; 4, 5, 6: observation holes; 7: lid.

\subsection{Dispersants}

The three tested dispersants (Yihe Co., Ltd) were microcrystalline cellulose, silicon dioxide, and polyethylene glycol. The ACL240 was divided into nine bottles, $20 \mathrm{~mL}$ each. The nine bottles were then divided into three groups, with each group having three different concentrations of dispersants $(0.5 \%, 0.75 \%$, and $1.0 \%)$. The samples containing microcrystalline cellulose were named A1, A2, and A3. The samples containing silicon dioxide were called B1, B2, and B3. The samples containing polyethylene glycol were named $\mathrm{C} 1, \mathrm{C} 2$, and $\mathrm{C} 3$.

\subsection{Particle size detection}

The equipment-Malvern Zetasizer Nano ZS (Malvern Panalytical, USA)-used to measure the particle size in this experiment is based on the principle of dynamic light scattering. When suspended particles undergo Brownian motion, they produce scattered stripes that change with time. The detector converts the scattered light signal into an electric current and obtains the diffusion coefficient of the particles in the solution through digital gas correlation. The size of the particle size can then be obtained using the Stock-Einstein equation. The equipment's particle size range falls within $0.6 \mathrm{~nm}$ to 6000 $\mathrm{nm}$, while the concentration range is $0.1 \mathrm{mg} / \mathrm{ml}$ to $40 \mathrm{wt} \%$, the temperature range is between $2{ }^{\circ} \mathrm{C}$ to $90^{\circ} \mathrm{C}$, and the LASER specification is $4 \mathrm{Mw}$ $\mathrm{He}-\mathrm{Ne}, 633 \mathrm{~nm}$. The nine samples were tested and recorded for particle size distribution each week.

\section{Results and discussions}

\subsection{Particle size distribution}

After 56 days of sampling and analysis, the average particle sizes of the different samples were summarized (Table 1). On the first day, the particle sizes were similar, but eventually they began to differ according to the effectiveness of the dispersants. The average particle sizes of the 
A1, A2 A3, B1, B2, B3, C1, C2, and C3 samples on the first day were $108.6 \mathrm{~nm}, 159.4 \mathrm{~nm}, 108.1$ $\mathrm{nm}, 123.7 \mathrm{~nm}, 116.2 \mathrm{~nm}, 176.3 \mathrm{~nm}, 131.1 \mathrm{~nm}$, $116.5 \mathrm{~nm}$, and $102.1 \mathrm{~nm}$, respectively. By the 56th day, the average particle sizes had changed to $667.1 \mathrm{~nm}, 709.4 \mathrm{~nm}, 234.4 \mathrm{~nm}, 558.3 \mathrm{~nm}$, $126.2 \mathrm{~nm}, 210.9 \mathrm{~nm}, 344.8 \mathrm{~nm}, 118.5 \mathrm{~nm}$, and $139.5 \mathrm{~nm}$, respectively. In the case of the A1 sample, the average particle size did not change much from the 1st day to the 28th day, but after the 42nd day, the aggregation degree began rising and eventually sky-rocketed to $667.1 \mathrm{~nm}$ by the 56th day. In the case of the A2 sample, the average particle size declined slightly in the first few weeks but then jumped to $709.4 \mathrm{~nm}$. In the B1 sample, the average particle size remained pretty stable in the first five weeks, but-as recorded on the 49th day-the particles started to re-aggregate soon thereafter. In the $\mathrm{C} 1$ sample, the average particle size increased quickly in the first week and was the only sample in which the average particle size was larger than 200. However, the average particle size of the A3, B2, B3, C2, and C3 samples was relatively stable over the 56 days, with the changes being negligible. After comparing each sample, it was found that $\mathrm{A} 3, \mathrm{~B} 2$, and $\mathrm{C} 2$ had the best dispersant effect, whereas $\mathrm{A} 2, \mathrm{~B} 1$ and $\mathrm{C} 1$ could not slow down the re-aggregation process of the mixture. As can be seen in Fig. 2, in the A1 sample, the proportion of particles above $1000 \mathrm{~nm}$ rocketed from $15 \%$ to $38 \%$ over the 56 days. Nevertheless, particles below $100 \mathrm{~nm}$ still accounted for $42 \%$ of the total, while particles in the range of $100 \mathrm{~nm}$ to $1000 \mathrm{~nm}$ dropped to $22 \%$ on the 56th day. The proportion of particles in the $200 \mathrm{~nm}$ to $1000 \mathrm{~nm}$ range in the $\mathrm{B} 1$ sample increased to $42.32 \%$ over the 56 days. At the same time, the proportion of particles with particle size below $100 \mathrm{~nm}$ dropped to $21.64 \%$, and particles below $50 \mathrm{~nm}$ disappeared completely. In the $\mathrm{C} 1$ sample, the proportion of particles in the $200 \mathrm{~nm}$ to $1000 \mathrm{~nm}$ range increased to $16.41 \%$ over the 56 days, with a sudden growth of particles above $500 \mathrm{~nm}$. In contrast, three samples-A3, B2, and C2-had the smallest average particle size and the most stable particle distribution compared with each other sample of the same group (Table 1), and thus the best dispersant effect. The proportion of particles in the $200 \mathrm{~nm}$ to $1000 \mathrm{~nm}$ range in the case of A3, B2, and $\mathrm{C} 2$ on the first day was $12.09 \%, 13.78 \%$, and $9.09 \%$, respectively. By the end of the eighth week, the numbers were $36,28 \%, 25.29 \%$, and $13.73 \%$, respectively (see Fig. 3).

Table 1. Average particle size of the different samples over 56 days

\begin{tabular}{cccccccc}
\hline Day & $\mathbf{1}$ & $\mathbf{7}$ & $\mathbf{2 8}$ & $\mathbf{3 5}$ & $\mathbf{4 2}$ & $\mathbf{4 9}$ & $\mathbf{5 6}$ \\
\hline Sample & & \multicolumn{7}{c}{ Average } & $\mathbf{7 2}$ particle size $\mathbf{( n m )}$ & \\
\hline A1 & 108.6 & 101.0 & 103.7 & 124.6 & 280.7 & 294.6 & 667.1 \\
A2 & 159.4 & 112.5 & 104.8 & 212.5 & 377.5 & 571.5 & 709.4 \\
A3 & 108.1 & 138.4 & 207.4 & 155.2 & 340.2 & 223.7 & 234.4 \\
B1 & 123.7 & 128.1 & 233.0 & 134.9 & 235.0 & 450.5 & 558.3 \\
B2 & 116.2 & 152.5 & 176.3 & 107.4 & 105.8 & 174.9 & 126.2 \\
B3 & 176.3 & 196.5 & 111.9 & 210.6 & 199.3 & 268.1 & 210.9 \\
C1 & 131.1 & 292.8 & 325.8 & 215.5 & 303.5 & 284.6 & 344.8 \\
C2 & 116.5 & 92.7 & 102.5 & 94.5 & 94.05 & 214.7 & 118.5 \\
C3 & 102.1 & 188.8 & 93.64 & 110.5 & 102.9 & 149.3 & 139.5 \\
\hline
\end{tabular}




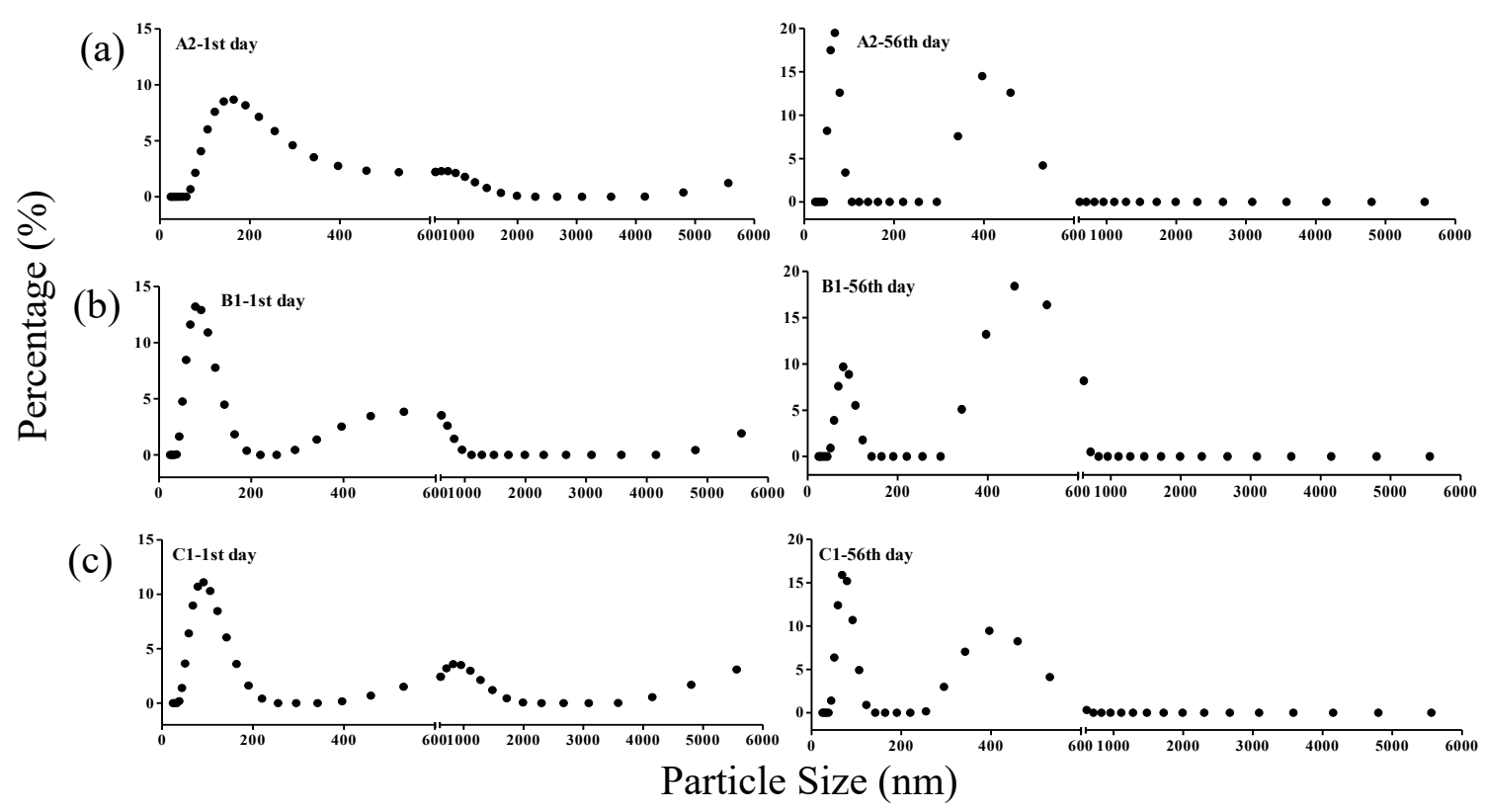

Figure 2. Particle size distribution diagram of the (a) A2 sample, (b) B1 sample, and (c) C1 sample on the 1 st day and the 56th day

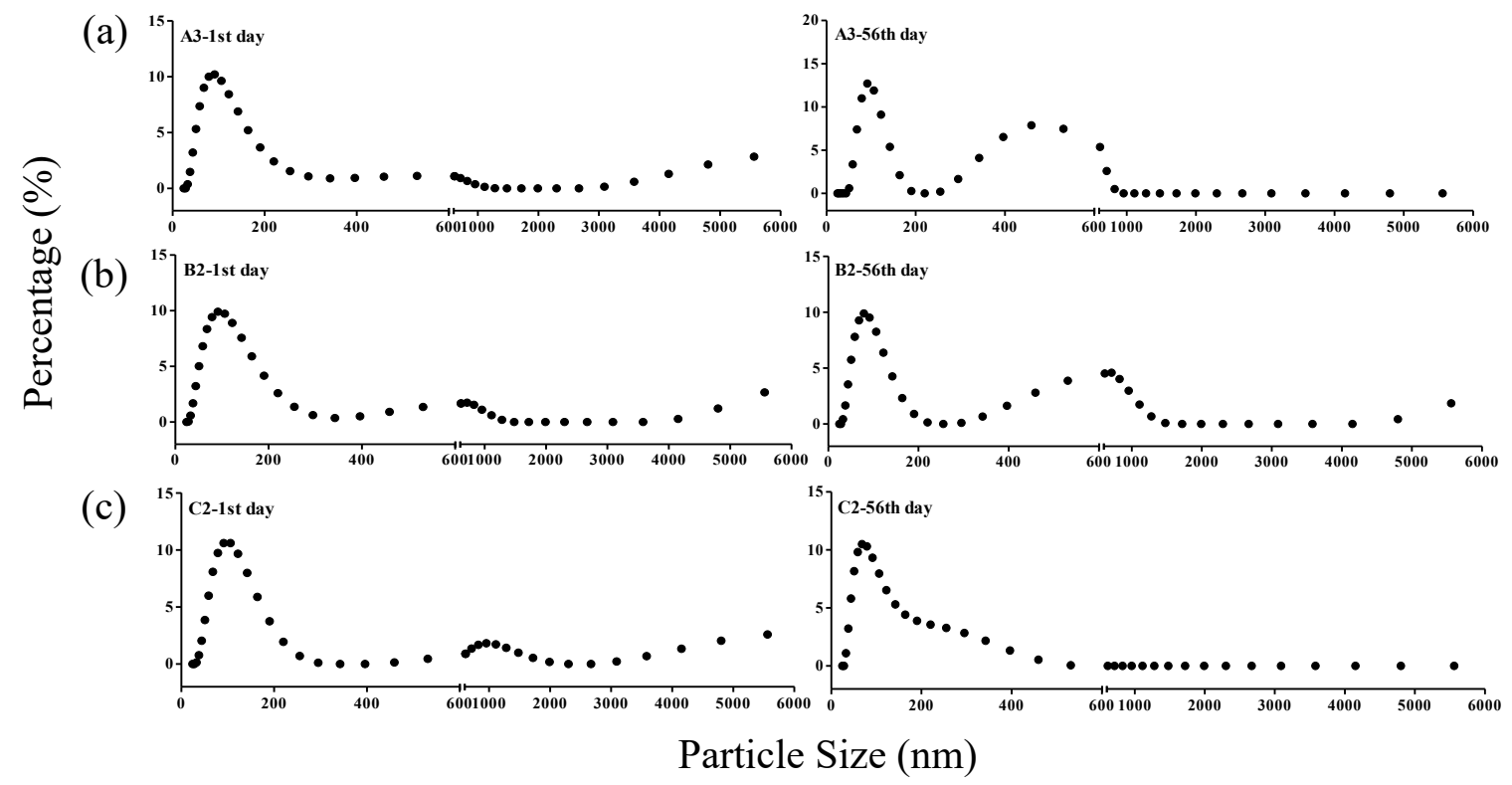

Figure 3. Particle size distribution diagram of the (a) A3 sample, (b) B2 sample, and (c) C2 sample on the 1 st day and the 56 th day

In order to evaluate the effectiveness of the chemical dispersants, in each sample, the particles whose sum demonstrated a positive growth over time were chosen. In the particles of the A1 sample [Fig. 4. (a)], the $531 \mathrm{~nm}$ and $1280 \mathrm{~nm}$ particles are negatively correlated. When the proportion of $531 \mathrm{~nm}$ particles decreases with time, the proportion of $1280 \mathrm{~nm}$ particles increases. Within eight weeks, the total proportion of $531 \mathrm{~nm}$ and $1280 \mathrm{~nm}$ particles increased to $13.51 \%$. After eight weeks, the 531 $\mathrm{nm}$ particles disappeared completely. In the A2 sample [Fig. 4. (b)], the $255 \mathrm{~nm}$ and $396 \mathrm{~nm}$ particles were chosen. The total proportion of the two particle sizes increased to $9.52 \%$, with the $255 \mathrm{~nm}$ particles disappearing totally by the 
eighth week. Among the particles in the A3 sample [Fig. 4. (c)], those that had a correlation were the $396 \mathrm{~nm}$ and $459 \mathrm{~nm}$ particles. Their total proportion rocketed from $1.99 \%$ to $14.41 \%$, with the proportion between the two particle sizes remaining stable during the eight weeks. In the B1 sample [Fig. 4. (d)], three particles sizes-396 nm, $459 \mathrm{~nm}$, and $531 \mathrm{~nm}$-had a positive rising trend. The total proportion of the three particles sizes increased to $48 \%$ by the eighth week. Among the particles in the B2 sample [Fig. 4. (e)], the $295 \mathrm{~nm}$ particles and the $825 \mathrm{~nm}$ particles had a negative correlation, with the total proportion of the two particle sizes increasing to $1.98 \%$. In the B3 sample [Fig. 4. (f)], a total of four particle sizes were reported to have had a positive growth over time. The sum of the $220 \mathrm{~nm}$ particles, the $255 \mathrm{~nm}$ particles, the $531 \mathrm{~nm}$ particles, and the $615 \mathrm{~nm}$ particles grew to $4.96 \%$ over eight weeks. Among the particles in the $\mathrm{C} 1$ sample [Fig. 4. (g)], the $342 \mathrm{~nm}$ particles and the $459 \mathrm{~nm}$ particles had a negative correlation, and over the eight weeks their total proportion increased to $14.41 \%$. In the $\mathrm{C} 2$ sample [Fig. 4. (h)], the proportion of the 220 $\mathrm{nm}$ particles and the $255 \mathrm{~nm}$ particles had a negative correlation. The total proportion of the two particle sizes rose to $4.18 \%$ after eight weeks. Among the particles in the C3 sample [Fig. 4. (i)], those that had a correlation were the $190 \mathrm{~nm}$ and the $531 \mathrm{~nm}$ particles. Their total proportion increased to $3.26 \%$ over the eight weeks.

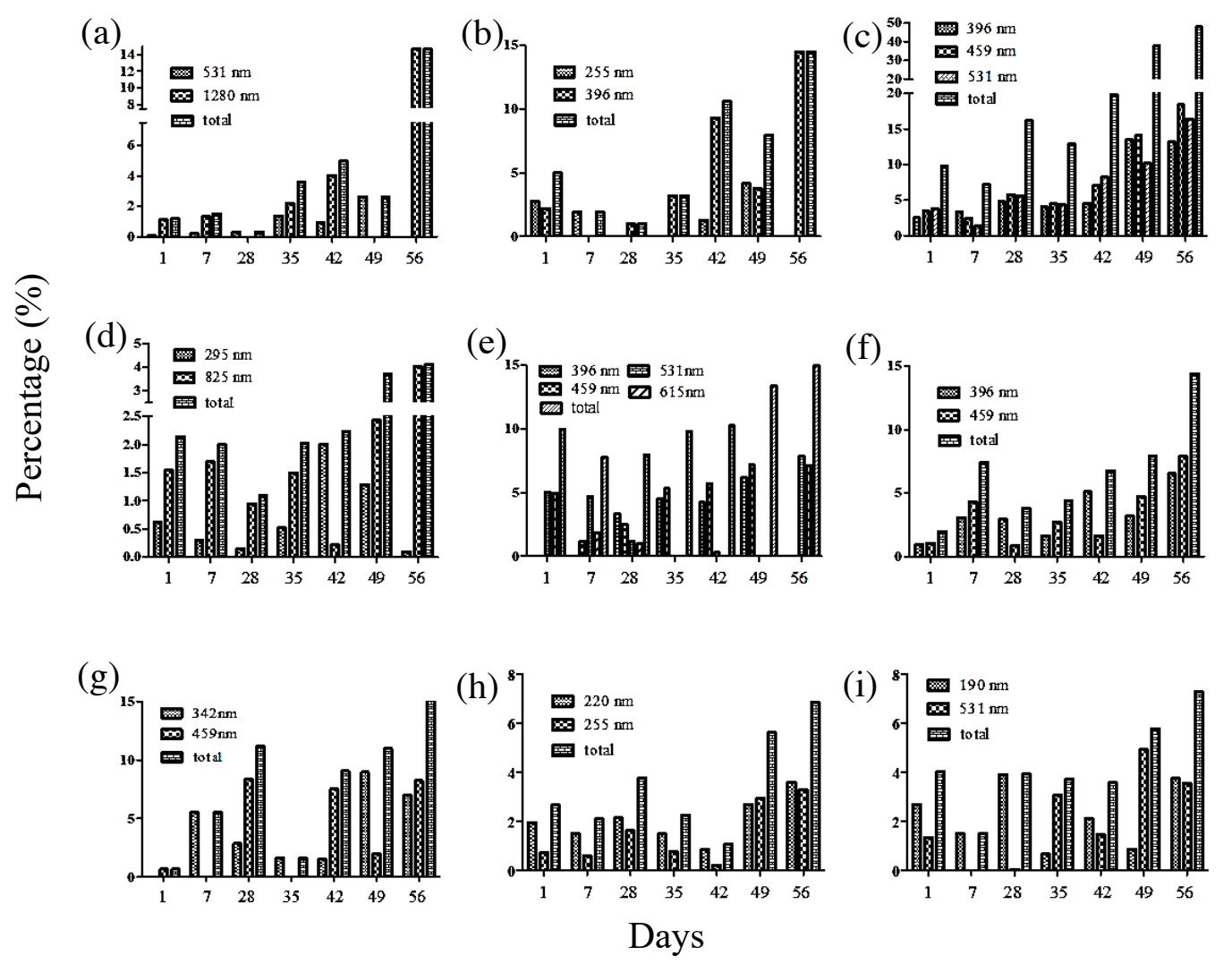

Figure 4. Histogram of the (a) $532 \mathrm{~nm}$ particles and $1280 \mathrm{~nm}$ particles in the A1 sample, (b) $255 \mathrm{~nm}$ particles and $396 \mathrm{~nm}$ particles in the A2 sample, (c) $396 \mathrm{~nm}$ particles and $459 \mathrm{~nm}$ particles in the A3 sample, (d) $396 \mathrm{~nm}$ particles, $459 \mathrm{~nm}$ particles, and $531 \mathrm{~nm}$ particles in the B1 sample, (e) $295 \mathrm{~nm}$ particles and $825 \mathrm{~nm}$ particles in the B2 sample, (f) $396 \mathrm{~nm}$ particles, $459 \mathrm{~nm}$ particles, $531 \mathrm{~nm}$ particles, and the $615 \mathrm{~nm}$ particles in the B3 sample, (g) $342 \mathrm{~nm}$ particles and $459 \mathrm{~nm}$ particles in the C1 sample, (h) $220 \mathrm{~nm}$ particles and $255 \mathrm{~nm}$ particles in the C2 sample, and (i) $190 \mathrm{~nm}$ particles and $531 \mathrm{~nm}$ particles in the B2 sample 


\subsection{Comparison of the particle sizes of the nine samples}

In the A1, A2 and A3 samples, the degree of aggregation at different concentrations varies with particles, aggregation and time. The stability of a sample was indicated by the value of the slope. When the slope value (or S) was large, the degree of aggregation was more obvious. In contrast, a value represented by $1 / \mathrm{S}$ meant that the larger the value, the more obvious the dispersion effect. The comparisons of the particle distribution slopes of each dispersant type were recorded as follows (Fig. 5). In the A1 sample, the total percentage of the $531 \mathrm{~nm}$ particles and the $1280 \mathrm{~nm}$ particles showed a positive correlation between aggregation and time. The sum of the $531 \mathrm{~nm}$ particles and the $1280 \mathrm{~nm}$ particles had a growing pattern. The degree of aggregation of the A1 sample was represented by the sum of the $531 \mathrm{~nm}$ particles and the $1280 \mathrm{~nm}$ particles, and the $1 / \mathrm{S}$ value was 6.32. In the A2 sample, the total percentage of the $255 \mathrm{~nm}$ particles and the $396 \mathrm{~nm}$ particles exhibited a positive correlation between aggregation and time. The total percentage changed and posted a growing trend over time. The degree of aggregation of the A2 sample was represented by the sum of the $255 \mathrm{~nm}$ particles and the $396 \mathrm{~nm}$ particles, and the 1/S value was 5.97. In the A3 sample, the total percentage of the $396 \mathrm{~nm}$ particles and the $459 \mathrm{~nm}$ particles indicated a positive correlation between aggregation and time. The sum of the $396 \mathrm{~nm}$ particles and the $459 \mathrm{~nm}$ particles posted a rising trend. Represented by the sum of the $396 \mathrm{~nm}$ particles and the $459 \mathrm{~nm}$ particles, the $1 / \mathrm{S}$ value of the A3 sample was 7.59. In conclusion, the $1 / \mathrm{S}$ values of the A1, A2, and A3 samples were 6.32, 5.97, and 7.59, respectively. In the B1 sample, the degree of aggregation of the $396 \mathrm{~nm}, 459 \mathrm{~nm}$, and $531 \mathrm{~nm}$ particles in total was the most obvious, and was therefore regarded as the representative. The $1 / \mathrm{S}$ value of the sum of the $396 \mathrm{~nm}, 459 \mathrm{~nm}$, and $531 \mathrm{~nm}$ particles in the B1 sample was 1.59 . In the B2 sample, the total percentage of the $295 \mathrm{~nm}$ particles and the 825 $\mathrm{nm}$ particles showed a positive correlation between aggregation and time. The sum of the $295 \mathrm{~nm}$ particles and the $825 \mathrm{~nm}$ particles posted a growing pattern. The degree of aggregation of the B2 sample was represented by the sum of the $295 \mathrm{~nm}$ particles and the $825 \mathrm{~nm}$ particles, and the $1 / \mathrm{S}$ value was 6.32 . The B3 sample was represented by the sum of the $220 \mathrm{~nm}$ and the 255 $\mathrm{nm}$ particles, together with the $531 \mathrm{~nm}$ and the $615 \mathrm{~nm}$ particles, and the $1 / \mathrm{S}$ value was 10.47 . In summary, the $1 / \mathrm{S}$ values of the B1, B2, and B3 samples were 1.59, 30.39, and 10.47, respectively. In the $\mathrm{Cl}$ sample, the total percentage of the $342 \mathrm{~nm}$ and the $459 \mathrm{~nm}$ particles showed a positive correlation between aggregation and time.
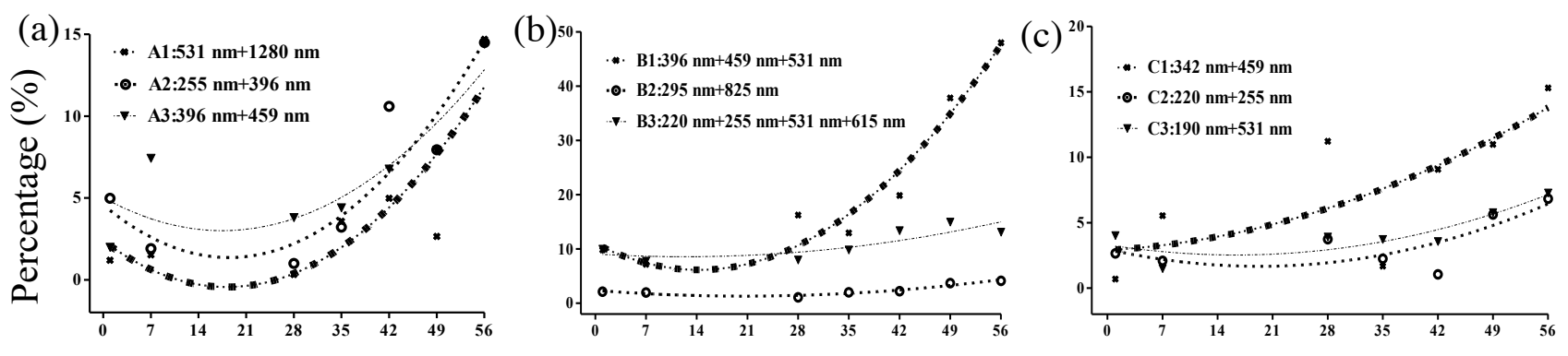

Days

Figure 5. Comparison of the particle distribution slopes of (a) A1, A2, and A3; (b) B1, B2, and B3; (c) $\mathrm{C} 1, \mathrm{C} 2$, and $\mathrm{C} 3$

The sum of the $342 \mathrm{~nm}$ and the $459 \mathrm{~nm}$ particles exhibited a growing pattern, and the $1 / \mathrm{S}$ value was 5.23. In the $\mathrm{C} 2$ sample, the total percentage of the $220 \mathrm{~nm}$ particles and the 255 $\mathrm{nm}$ particles showed a positive effect on the relationship between aggregation and time. The sum of the $220 \mathrm{~nm}$ particles and the $255 \mathrm{~nm}$ particles posted a growing pattern. The C2 sample was represented by the sum of the 220 $\mathrm{nm}$ particles and the $255 \mathrm{~nm}$ particles, and the $1 / \mathrm{S}$ value was 17.32 . In the $\mathrm{C} 3$ sample, the total percentage of the $190 \mathrm{~nm}$ and the $531 \mathrm{~nm}$ 
particles posted a positive correlation between aggregation and time. The sum of the $190 \mathrm{~nm}$ and the $531 \mathrm{~nm}$ particles showed a growing pattern. The C3 sample was represented by the sum of the $190 \mathrm{~nm}$ and the $531 \mathrm{~nm}$ particles, and the $1 / \mathrm{S}$ value was 15.36 . The $1 / \mathrm{S}$ values of $\mathrm{C} 1, \mathrm{C} 2$, and C3 were 5.23, 17.32, and 15.36, respectively.

\section{Conclusions}

Nanoparticles are affected by the Van der Waals force regardless of what dispersant is used, because as the particle size decreases, the surface area increases. As time passes, the particles are easily re-aggregated into macromolecules. Therefore, dispersant addition is necessary in order to maintain the stability of the micronized extract. After monitoring and testing for the particle size distribution over eight weeks, the results showed that the degree of aggregation did not decrease when the amount of dispersant was increased, but rather the optimal amount worked better.

\subsection{Particle size distribution}

All the micro-particles displayed an aggregation trend into bigger particles. Among the nine samples, the A1 and A2 samples had the most obvious degree of aggregation. The particles ranging from $100 \mathrm{~nm}$ to $1000 \mathrm{~nm}$ in the A1 sample aggregated into particles above 1000 $\mathrm{nm}$. In the A2 samples, most of the particles ranged in size from $200 \mathrm{~nm}$ to $1000 \mathrm{~nm}$, and by the 56th day, particles below $50 \mathrm{~nm}$ no longer existed. In contrast, three samples performed the best in terms of slowing the aggregation process, namely A3, B2, and C2 (Fig. 6). In the A3 sample, the particles ranging in size from $200 \mathrm{~nm}$ to $1000 \mathrm{~nm}$ had a slight change over time, but the degree of aggregation in this sample was lower than in the other samples of the same group. The particle size in the B2 sample was stable during the 56 days of the test, and no remarkable changes were recorded. Moreover, the degree of aggregation even slowed down in the last week. In the $\mathrm{C} 2$ sample, the $200 \mathrm{~nm}-1000 \mathrm{~nm}$ range particles increased stably over the first six weeks, but accelerated quickly in the seventh week and then dropped.

\subsection{Comparison of the particle distribution slopes of the three dispersant representatives}

The degree of aggregation differed by particle, aggregation and time in each sample with different concentrations.

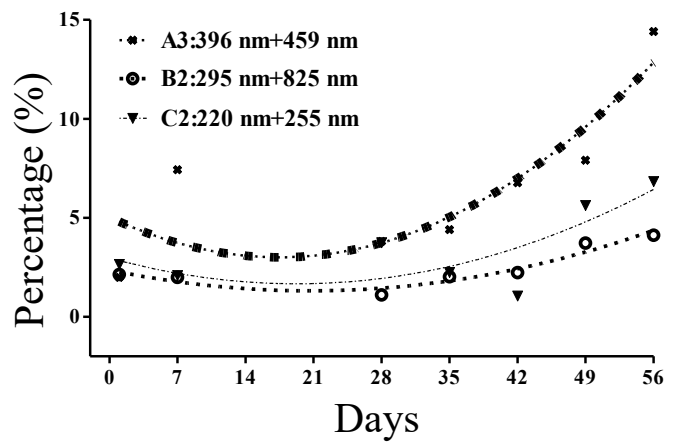

Figure 6. Comparison of the particle distribution slopes of A3, B2, and C2

The slope value (a.k.a. S) represented the degree of aggregation of the samples, with the larger the $1 / \mathrm{S}$, the more obvious the dispersion effect. Each sample had a corresponding set of particles, which represented a positive effect on the relationship between aggregation and time, and thus a growing pattern. The different concentrations of each dispersant were compared and chosen for the best, and each representative was then compared for the best dispersant out of the three. The $1 / \mathrm{S}$ values of $\mathrm{A} 1$, $\mathrm{A} 2$, and A3 were 6.32, 5.97, and 7.59, respectively, meaning that microcrystalline cellulose $1.0 \%$ had the best dispersion effect. The $1 / \mathrm{S}$ values of $\mathrm{B} 1, \mathrm{~B} 2$, and $\mathrm{B} 3$ were 1.59 , 30.39 , and 10.47 , respectively, meaning that silicon dioxide $0.75 \%$ had the best dispersion effect. The $1 / \mathrm{S}$ values of $\mathrm{C} 1, \mathrm{C} 2$, and $\mathrm{C} 3$ were $5.23,17.32$, and 15.36, respectively, meaning that polyethylene glycol had the best dispersion effect at a concentration of $0.75 \%$. Figure 6 compares the three particle distribution slopes and indicates the best one out of the three. The A3 particles aggregated faster than the B2 particles, and the aggregation speed of the $\mathrm{C} 2$ particles was also faster than that of the B2 particles. Hence, a silicon dioxide concentration of $0.75 \%$ gives the best dispersion effect. 


\section{References}

Alwadani, N., Fatehi, P. (2018). Synthetic and lignin-based surfactants: challenges and opportunities. Carbon Resources Conversion, 1, 126-138.

Chang, C.Y., Lee, C.L., Pan, T.M. (2006). Statistical optimization of medium components for the production of Antrodia cinnamomea AC0623 in submerged cultures. Applied Microbiology Biotechnology, 72, 654-661.

Cha, W.S., Ding, J.L., Choi, D. (2009). Comparative evaluation of antioxidant, nitrite scavenging, and antitumor effects of Antrodia camphorata extract. Biotechnology and Bioprocess Engineering, 14, 232-237.

Fan, H., Lu, F., Yang, A., Dong, Y., Liu, P., Wang, Y. (2019). A review on the nonpharmacological therapy of traditional chinese medicine with antihypertensive effects. Evidence-Based Complementary and Alternative Medicine, 2019, 1-8.

Guo, Z., Martucci, N.J., Liu, Y., Yoo, E., Tako, E., Mahler, G.J. (2018). Silicon dioxide nanoparticle exposure affects small intestine function in an in vitro model. Nanotoxicology, 12, 485-508.

Geethangili, M., Tzeng, Y.M. (2011). Review of pharmacological effects of Antrodia camphorata and its bioactive compounds. Evidence-Based Complementary and Alternative Medicine, 2011, 1-17.

Hadar, Y., Dosoretz, C.G. (1991). Mushroom mycelium as a potential source of food flavor. Trends in Food Science and Technology, 2, 214-218.

Lee, I.H., Huang, R.L., Chen, C.T., Chen, H.C., Hsu, W.C., Lu, M.K. (2002). Antrodia camphorata polysaccharides exhibit antihepatitis B virus effects. FEMS Microbiology Letters, 209(1), 63-67.

Lin, E.S., Wang, C.C., Sung, S.C. (2006). Cultivating conditions influence lipase production by the edible Basidiomycete Antrodia cinnamomea in submerged culture. Enzyme and Microbial Technology, 39(1), 98-102.
Laszczyk, M.N. (2009). Pentacyclic Triterpenes of the Lupane, Oleanane and Ursane Group as Tools in Cancer Therapy. Planta medica, 75(15), 1549-1560.

Lin, Y.S., Cheng, W.C., Chen, S.H., Yeh, A.I. (2012). Preparation and characterization of nano/submicron particles of ganoderma lucidum fruiting body. Journal of Food and Drug Analysis, 20(4), 900-907.

Mikaela Börjesson (2015). Crystalline Nanocellulose - Preparation, Modification, and Properties Cellulose - Fundamental Aspects and Current Trends. Matheus Poletto, 159-191.

Sone, Y., Okuda, R., Wada, N., Kishida, E., Misaki, A. (2014). Structures and antitumor activities of the polysaccharides isolated from fruiting body and the growing culture of mycelium of Ganoderma Lucidum. Agricultural and Biological Chemistry, 49(9), 2641-2653.

Tang, J.I., Sun, J., He, Z.G. (2007). Selfemulsifying drug delivery systems: strategy for improving oral delivery of poorly soluble drugs. Current Drug Therapy, 2(1), 85-93.

Tzeng, Y.M., Geethangili, M., Ling, S.T. (2011). Purification of bioactive compounds from Antrodia camphorata and their pharmacological activities. Journal of Bioscience and Bioengineering, 108, s23.

Vidyasagar, C.C., Arthoba Naik, Y. (2016). Surfactant (PEG 400) effects on crystallinity of $\mathrm{ZnO}$ nanoparticles. Arabian Journal of Chemistry, 9(4) 507-510.

Xu, Y.H., Wang, N.S. (2004). Review and analysis of present status of the micronization of Chinese traditional medicine. China Journal of Chinese Materia Medica, 29(6), 497-500.

Zapadka, K.L., Becher, F.J., Gomes dos Santos, A.L., Jackson, S.E. (2017). Factors affecting the physical stability (aggregation) of peptide therapeutics. Interface Focus, 7(6), $1-18$.

\section{Acknowledgment}

This study was funded in part by MU Research Project 2020. 